PRAXISBEISPIEL

\title{
Optimierungspotenzial in der Lohnbuchhaltung
}

Volker Schmidt

Die Suche nach Rationalisierungspotenzialen in sozialen Unternehmen beginnt nicht bei den klientennahen Leistungen, sondern in der Verwaltung. Und auch innerhalb der Verwaltung sollten zuerst diejenigen Leistungsprozesse untersucht werden, deren Rationalisierung für die Lebensqualität der Klienten keine spürbaren Auswirkungen haben. Eine Möglichkeit für viele Träger bietet die Optimierung der Lohnbuchhaltung.

Empirische Hinweise auf Einsparmöglichkeiten in der Lohnbuchhaltung liefert eine jüngst erschienene Untersuchung der Kanzlei Lohhoff \& Partner. An dieser Studie mit dem Titel »Personalabrechnungsprozesse - Prozesse IT - Strukturen - Kostenpotenziale« waren sieben Unternehmen aus verschiedenen Branchen und mit unterschiedlichen Größen (zwischen 270 und 12.000 Mitarbeitern) beteiligt. Bei den untersuchten Unternehmen waren sowohl PC- als auch rechenzentrumsorientierte Softwareprodukte im Einsatz. Analysiert wurden die Personalkosten, Hard- und Softwarekosten, gegebenenfalls Kosten für einen externen Abrechnungsdienstleister und sonstige Kosten für Druck, Kuvertierung und Versand der Abrechnungen und Meldungen (vgl. Grafik 1).

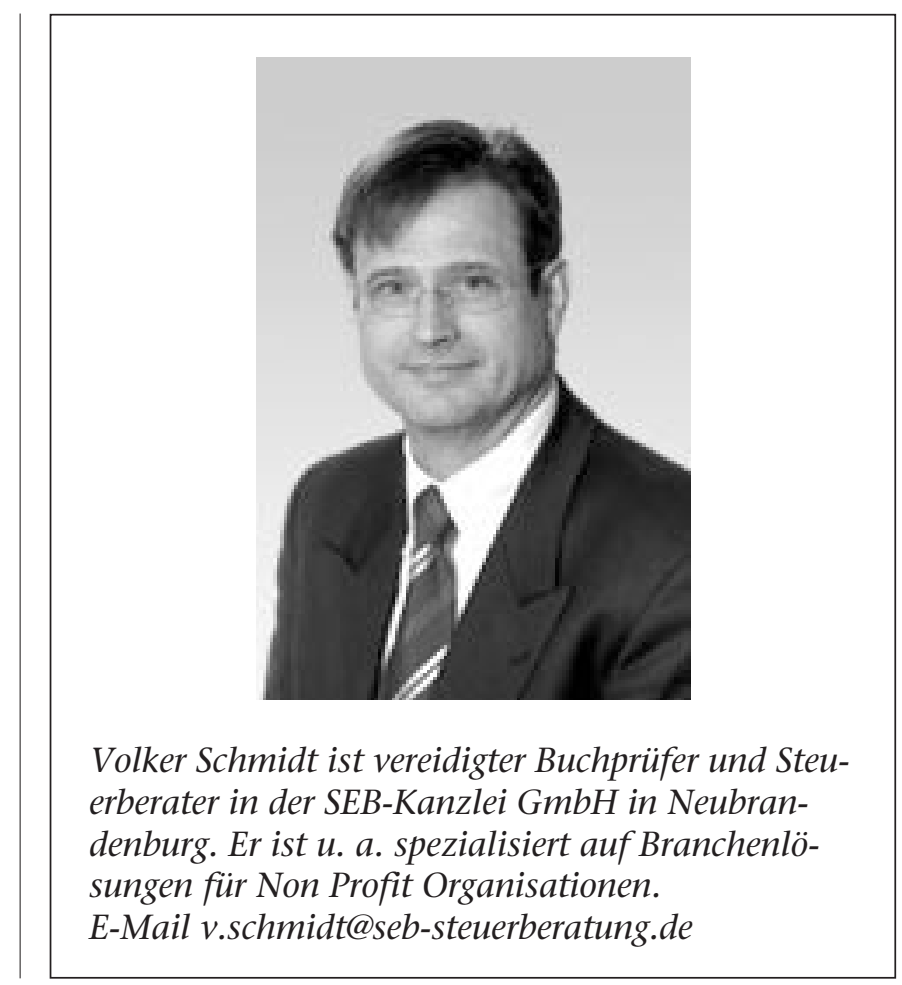

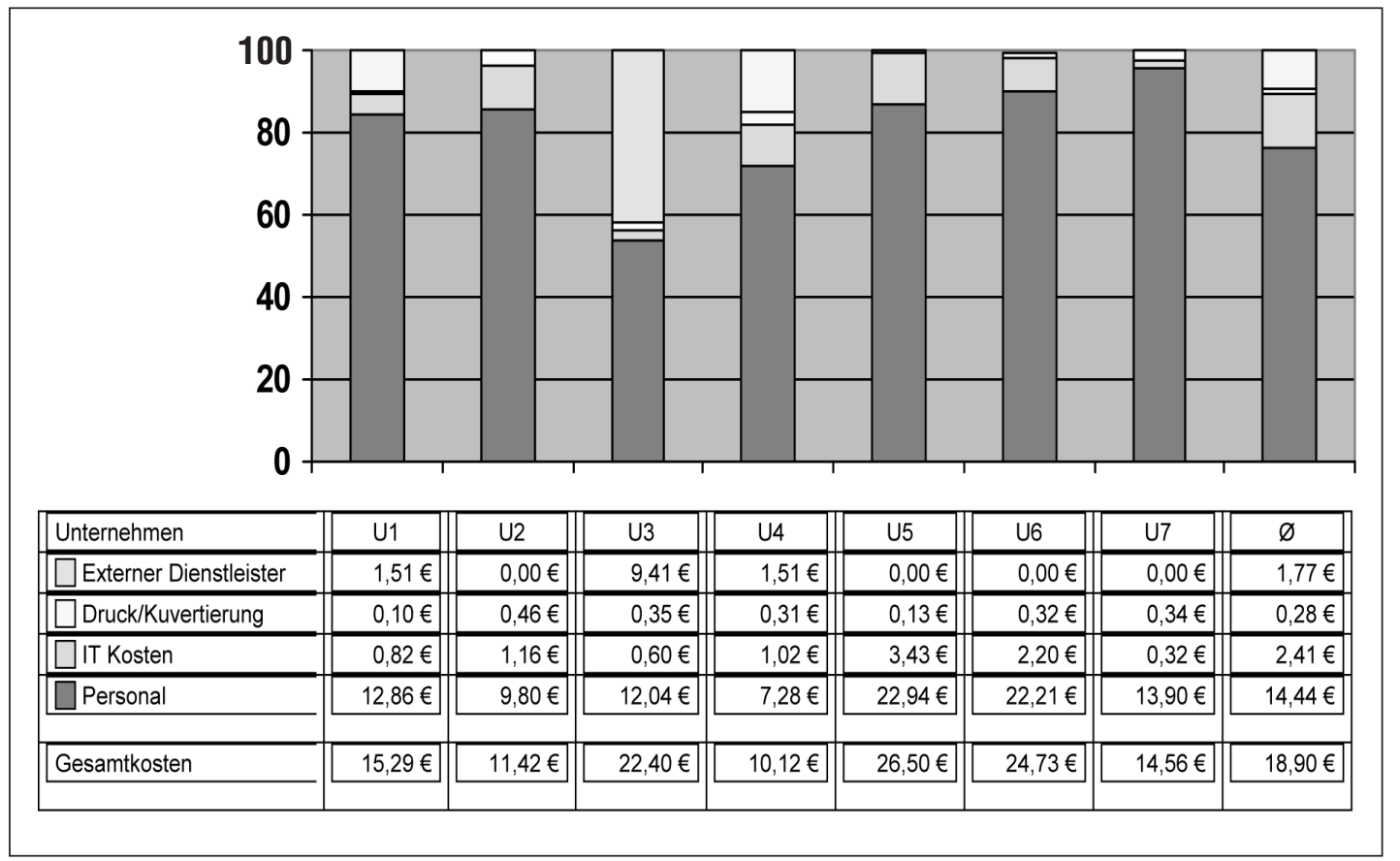

Grafik 1: Einsparpotenziale in sieben Unternehmen verschiederer Branchen und Größe 
Als Ergebnis zeigte sich deutlich, dass die Personalkosten den entscheidenden Faktor darstellen. Die Personalkosten erklären im Durchschnitt 80 Prozent der gesamten Kosten der Lohnbuchführung. Noch interessanter als dieses Resultat sind jedoch die Hinweise auf Rationalisierungspotenziale und entsprechende Kostensenkungen. In erster Linie durch konsequente Prozessautomatisierung und Prozessoptimierung sind Einsparpotenziale von nachweislich bis zu 50 Prozent zu erzielen.

\section{Rationalisierungspotenziale erkennen}

Deutlich niedrigere Prozesskosten werden insbesondere durch eine möglichst papierarme Abwicklung erreicht. Das zeitaufwendige Papierhandling wie Sortieren und Ablegen von Auswertungen, Zusammenstellen, Kuvertieren und Versendungen der Auswertungen kann komplett entfallen, wenn der Geschäftsprozess Lohnbuchhaltung durch Automatisierung optimiert wird.

Doch es können auch dann spürbare Erfolge erzielt werden, wenn nur einzelne Teilprozesse verändert werden. Um kostenrelevante Potenziale aufspüren zu können, sollte der Lohnprozess mit einem Analysetool durchleuchtet werden. (1)

Auswertungen verdeutlichen, in welchen Lohnprozessschritten die Träger noch bisher ungenutzte Möglichkeiten haben (vgl. Grafik 2). Checklisten und Demos zeigen, wie Funktionen des »papierlosen Lohnprozesses « konkret eingesetzt werden können, um Bearbeitungszeiten einzusparen.

\section{Outsourcing prüfen}

Auch mit dem gewonnenen Wissen über Optimierungspotenziale bleibt weiterhin die Frage bestehen, ob der
Lohnprozess nicht insgesamt ausgegliedert werden sollte, weil dann im Betrieb lediglich der Zeitaufwand zur Feststellung der Ein- und Austritte und übrigen laufenden Veränderungen anfällt. Neben dem klassischen Effekt von Outsourcing, fixe Kosten in variable Kosten umgewandelt zu haben, bietet eine Auslagerung des Lohnprozesses einige Vorteile:

- verminderte IT-Kosten

- Unabhängigkeit von Urlaub, Krankheit und Fortbildungen

- Rückfragen der Kassen, Ämter und gegebenenfalls der Mitarbeiter werden vom Dienstleister übernommen

- vollständige Entlastung von Bescheinigungen und Statistiken

- Betriebsprüfungen werden im Wesentlichen außer Haus und ohne Nachzahlungsrisiken abgewickelt

- Hotlinefunktion

Andererseits tritt in der Einrichtung kein Informationsdefizit ein. Die Daten des Lohnprozesses können per CD-ROM als Auskunftssystem auf der eigenen Anlage installiert werden.

Ob sich konsequentes Outsourcing oder eine eher behutsamere interne Rationalisierung der Lohnbuchhaltung anbietet, lässt sich erst nach einer Analyse des Lohnprozesses entscheiden. Doch als erster Anhaltspunkt können Ergebnisse eigener Projekte dienen. Abrechnungspreise liegen bei kleineren Einrichtungen bei 6,90 Euro und bei größeren Einrichtungen bei 6,00 Euro. Diese Zielkosten bilden die Benchmark.

\section{Anmerkungen}

(1) Die folgenden Ausführungen basieren auf dem »Analysetool Lohnbuchhaltung « der Steuerberatungsfirma SEB GmbH.

(2) Angaben über Referenzmodelle der Steuerberatungsfirma SEB $\mathrm{GmbH}$ erhalten Sie gerne vom Verfasser. Für Projekte im sozialen Bereich besteht eine Kooperation der SEB GmbH mit der Professur »Management in sozialen Einrichtungen/OE« (Prof. Dr. Bernd Halfar) an der Katholischen Universität Eichstätt-Ingolstadt.

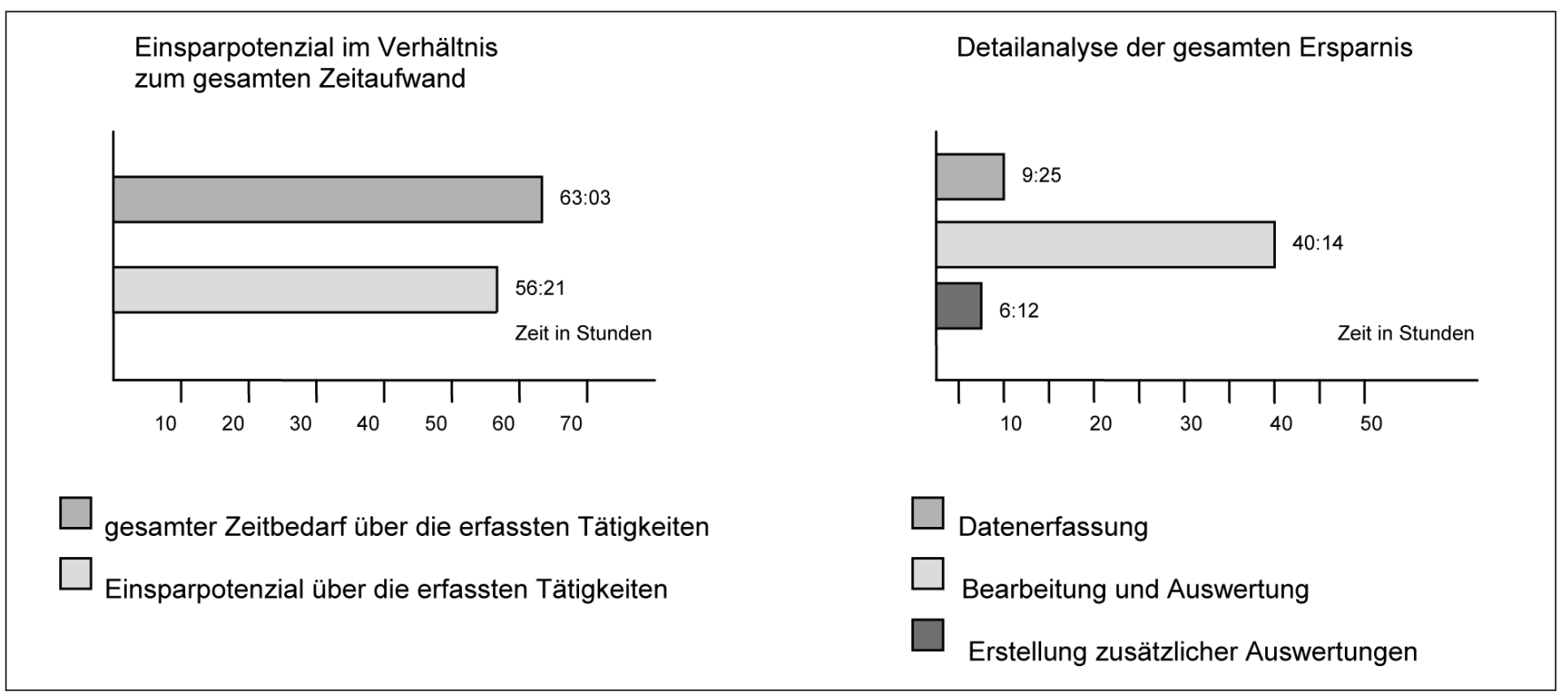

